\title{
Anti-infective treatment of purulent meningitis caused by carbapenem-resistant Klebsiella pneumoniae in a newborn: a case report
}

\author{
Faqin Wang ${ }^{1 \#}$, Juan Jiang ${ }^{1 \#}$, Guoqin Shi' ${ }^{2}$ Junyan Wang ${ }^{3}$, Suqin Zhou ${ }^{1}$ \\ ${ }^{1}$ Department of Clinical Pharmacy, Lanzhou University Second Hospital, Lanzhou, China; ${ }^{2}$ Department of Pharmacy, The Second People's Hospital \\ of Lanzhou City, Lanzhou, China; ${ }^{3}$ Neonatal Intensive Care Unit, Lanzhou University Second Hospital, Lanzhou, China \\ \#These authors contributed equally to this work. \\ Correspondence to: Suqin Zhou, MM. Department of Clinical Pharmacy, Lanzhou University Second Hospital, Lanzhou 730030, China. \\ Email: katherinesuqin@163.com.
}

\begin{abstract}
The widespread use of carbapenems has caused a notable spread of carbapenem-resistant Klebsiella pneumoniae (CRKP). The incidence of CRKP-associated infections is rising significantly in neonatal intensive care units (NICUs), which poses a grave challenge to clinical treatment. This paper is to highlight the drug treatment of CRKP with purulent meningitis in children and explore the safety of levofloxacin in children. We retrospectively analyzed the clinical data of combination therapy with levofloxacin and aztreonam in a newborn with purulent meningitis caused by CRKP. As clinical pharmacists, we evaluated the risks and benefits of quinolones for anti-infective treatment in newborns, helped clinicians adjust the antiinfective protocol of levofloxacin combined with aztreonam and provided pharmaceutical care throughout the course of treatment. In the end, the child had no fever, no dyspnea, and no obvious abnormalities in brain color Doppler ultrasound. The intracranial infection was finally controlled, and the child improved and was discharged, with no apparent neurological, skeletal, joint, tendon, or cardiac adverse events. For newborns with CRKP-associated purulent meningitis, fluoroquinolones combined with other drugs such as polymyxin, tigecycline, aminoglycosides, minocycline, that Klebsiella pneumoniae is susceptible to (when no safe and effective anti-infective alternatives are available) can reduce the mortality rate of newborns with purulent meningitis caused by carbapenem-resistant gram-negative bacteria. We analyzed the drug resistance mechanisms of CRKP, the selection of antibiotic agents, the safety of quinolones in children, the permeability of the blood-brain barrier to quinolones, and the selection of the quinolone dose. Personalized combination therapy improves treatment outcomes and reduces adverse reactions, especially in patients with resistant bacteria infection.
\end{abstract}

Keywords: Case report; levofloxacin; carbapenem-resistant Klebsiella pneumoniae (CRKP); purulent meningitis; newborn

Submitted Jul 15, 2020. Accepted for publication Oct 12, 2020.

doi: $10.21037 /$ tp-20-296

View this article at: http://dx.doi.org/10.21037/tp-20-296

\section{Introduction}

Bacterial meningitis is more common in the first month than at any other time of life. Despite advances in infant intensive care, neonatal meningitis remains a devastating disease. Group B Streptococcus (GBS), Escherichia coli, and other gram-negative bacilli are the most common causes of neonatal purulent meningitis. The risk factors for neonatal purulent meningitis include low birth weight (birth weight $<2,500 \mathrm{~g}$ ), premature delivery ( $<37$ weeks of gestation), premature rupture of membranes, septic or traumatic delivery, fetal birth Hypoxia, perinatal infection of mother, galactosemia, etc. (1). 
In recent years, the widespread use of carbapenems has caused a notable spread of carbapenem-resistant Klebsiella pneumoniae (CRKP). The incidence of CRKP-associated infections is rising significantly in neonatal intensive care units (NICUs), which poses a grave challenge to clinical treatment. The 28-day mortality rate of CRKP-associated infections is as high as $40 \%$ (2). To treat CRKP-associated infections, clinicians may use quinolones combined with other drugs based on drug susceptibility tests, but the safety effectiveness, and pharmacokinetics of quinolones as an anti-infective treatment in children are not well known, especially in newborn. With central nervous system infections, how well antibacterial agents cross the bloodbrain barrier to reach the cerebrospinal fluid determines the treatment outcome. This paper is to highlight the drug treatment of CRKP with purulent meningitis in children and explore the safety of levofloxacin in children. It is difficult to make a rational treatment strategy, the guides recommend some drugs in the program are not recommended for children, especially in newborn, such as polymyxin, tigecycline, aminoglycosides, sulfa drugs, etc., In this paper, we will analyze and discuss the case data of combination therapy with levofloxacin and aztreonam in a newborn with CRKP-associated purulent meningitis and perform a literature review of recent studies on the use of quinolones for anti-infective treatment in children in order to provide new ideas for rational drug use in children.

We present the following article in accordance with the CARE reporting checklist (available at http://dx.doi. org/10.21037/tp-20-296).

\section{Case presentation}

A 22-day-old girl weighing $3.5 \mathrm{~kg}$ was admitted to the local hospital due to "kin rash for 2 days and fever for 1 day". The girl was born to a gravida 1 para 1 (G1P1) woman via vaginal route at a gestational age of 39 weeks and 6 days. During labor, the amniotic fluid was clear, with no premature rupture of membranes. Her parents were healthy. Two days before admission, the patient had a rash on her face, which grew and spread to her entire body on the next day. On the night before admission, she had a fever up to $38{ }^{\circ} \mathrm{C}$. Physical examination and laboratory tests showed capillary refill time (CRT) $<3$ seconds, blood pressure (BP) 54/38 mmHg, C-reactive protein (CRP) $25.1 \mathrm{mg} / \mathrm{L}$, and procalcitonin (PCT) $0.33 \mathrm{ng} / \mathrm{mL}$. She was given cefoperazone sodium and sulbactam sodium, deslanoside (Cedilanide), dopamine, dobutamine, and epinephrine. However, the patient had convulsions (limb twitching), which improved after phenobarbital treatment. She was referred to and admitted to our hospital due to persistent fever.

Physical examination at admission showed temperature $38^{\circ} \mathrm{C}$, pulse $162 \mathrm{bpm}$, respiratory rate 56 breaths per minute, BP 133/91 mmHg, oxygen saturation $96 \%$ without oxygen therapy, body length $54 \mathrm{~cm}$, head circumference $35.5 \mathrm{~cm}$, chest circumference $36 \mathrm{~cm}$, fair nutritional status, conscious, poor mental response, mottled skin, and blood oozing in the umbilical area. Blood tests showed white blood cell (WBC) count $12.8 \times 10^{9} / \mathrm{L}$, neutrophils $61 \%$, lymphocytes $23 \%$, hemoglobin $129 \mathrm{~g} / \mathrm{L}$, platelet (PLT) count $184 \times 10^{9} / \mathrm{L}$, CRP $58.4 \mathrm{mg} / \mathrm{L}$, and PCT $0.812 \mathrm{ng} / \mathrm{mL}$. Both blood and cerebrospinal fluid cultures were negative for bacterial growth. Routine analysis of cerebrospinal fluid showed that Pandy's test was negative and that the WBC count in body fluids (WBC-BF) was $6.0 \times 10^{6} / \mathrm{L}$. Rapid biochemical analysis of cerebrospinal fluid showed a serum albumin (ALB) of $0.78 \mathrm{~g} / \mathrm{L}$, glucose (GLU) of $2.8 \mathrm{mmol} / \mathrm{L}$, and chloride (CL) of $115.2 \mathrm{mmol} / \mathrm{L}$. Transcranial color Doppler ultrasound showed a small amount of bilateral subependymal bleeding (absorption stage). Diagnoses at admission included fever, sepsis, convulsions, purulent meningitis.

After admission, the patient was given intermittent nasal oxygen therapy, heat (to keep her warm), rehydration, intravenous nutrition, nasal feeding, anti-infective treatment. Starting on day 1 after admission, she was given anti-infective treatment with meropenem $0.07 \mathrm{~g}(20 \mathrm{mg} / \mathrm{kg})$, ivgtt, $\mathrm{q} 8 \mathrm{~h}$, running from days 1 to 8 . On day 3 after admission, the county hospital reported positive blood culture (cocci), but they did not perform drug susceptibility tests. The patient still had intermittent fever (up to $39^{\circ} \mathrm{C}$ ), and vancomycin was added at the dose of $0.04 \mathrm{~g}(10 \mathrm{mg} / \mathrm{kg})$, ivgtt, $\mathrm{q} 8 \mathrm{~h}$ from days 3 to 8 . On day 9 after admission, systemic rashes, mainly on the face and chest, worsened. The rashes were red and bulging, some of them merged into patches, and some broke. Herpes simplex was considered. Meropenem and vancomycin were discontinued, and antiviral therapy [acyclovir $0.06 \mathrm{~g}(15 \mathrm{mg} / \mathrm{kg})$, ivgtt, $\mathrm{q} 8 \mathrm{~h}$ ] and anti-allergic therapy [methylprednisolone sodium succinate $4 \mathrm{mg}(1 \mathrm{mg} / \mathrm{kg})$, ivgtt, qd] were given from days 9 to 16 . On day 12 after admission, the patient had apnea and was given invasive ventilation in nasal continuous positive airway pressure (nCPAP) mode from days 12 to 13. Transcranial color Doppler ultrasound showed a small amount of bilateral subependymal bleeding (mostly 
absorbed). On day 16 after admission, the patient still had intermittent fever (up to $38.6^{\circ} \mathrm{C}$ ). Next-generation sequencing (NGS) of cerebrospinal fluid showed Klebsiella pneumoniae. Sputum culture was positive for CRKP, which was susceptible only to aztreonam, aminoglycosides, and fluoroquinolones per the drug susceptibility tests. Routine analysis of cerebrospinal fluid showed that Pandy's test was weakly positive and that $\mathrm{WBC}-\mathrm{BF}$ was $22.0 \times 10^{6} / \mathrm{L}$. Rapid biochemical analysis of cerebrospinal fluid showed PROT of $2.14 \mathrm{~g} / \mathrm{L}, \mathrm{GLU}$ of $2.3 \mathrm{mmol} / \mathrm{L}$, and CL of $116.3 \mathrm{mmol} / \mathrm{L}$. Transcranial color Doppler ultrasound was unremarkable.

Taking all of the above together, we diagnosed the patient with purulent meningitis. Acyclovir was discontinued, and the patient was given aztreonam $0.11 \mathrm{~g}(30 \mathrm{mg} / \mathrm{kg})$, ivgtt, $\mathrm{q} 8 \mathrm{~h}$ and levofloxacin $0.04 \mathrm{~g}(10 \mathrm{mg} / \mathrm{kg})$, ivgtt, $\mathrm{qd}$ from days 16 to 32, after obtained the parents' consent (signed informed consent). During the treatment, the patient had intermittent, involuntary, asymmetric limb twitching that lasted several seconds to tens of seconds per episode. Therefore, she was given oral levetiracetam solution $0.4 \mathrm{~mL}(10 \mathrm{mg} / \mathrm{kg})$, po, bid from days 16 to 38 . By day 25 after admission, fever, rash, and limb twitching were alleviated; transcranial color Doppler ultrasound was unremarkable; routine analysis of cerebrospinal fluid showed that Pandy's test was positive and that WBC-BF was $3.0 \times 10^{6} / \mathrm{L}$; rapid biochemical analysis of cerebrospinal fluid showed PROT of $0.95 \mathrm{~g} / \mathrm{L}$, GLU of $2.2 \mathrm{mmol} / \mathrm{L}$, and $\mathrm{CL}$ of $117.3 \mathrm{mmol} / \mathrm{L}$ (improved from before). Moreover, the patient received acupuncture to alleviate limb twitching. The patient also received training to improve swallowing disorders, gross motor function, and language function. On day 32 after admission, her mental state had improved, and her fever had abated. She still had occasional limb twitching, with a small amount of scattered rash on her face. She had no shortness of breath or difficulty breathing (no oxygen therapy). Transcranial color Doppler ultrasound was unremarkable, and levofloxacin and aztreonam were discontinued.

The patient had no apparent adverse drug events during hospitalization. Clinicians will follow-up this patient to monitor common, expected, and potential adverse events, such as rash, diarrhea, and potential musculoskeletal or neurological events.

The Figure 1 shows the timeline of drug treatment. We have obtained the parents' consent to publish this case report and all related results and images in academic journals. All procedures performed in studies involving human participants were in accordance with the ethical standards of the institutional and/or national research committee(s) and with the Helsinki Declaration (as revised in 2013).

\section{Discussion}

The diagnostic criteria for purulent meningitis is that bacterial pathogens isolated from cerebrospinal fluid culture can be diagnosed as purulent meningitis. The most common clinical signs of neonatal purulent meningitis are unstable body temperature, irritability or lethargy, feeding difficulties or vomiting, bulging fontanelle, and rigid neck (3). Take include abnormal cerebrospinal fluid test results, such as increased cerebrospinal fluid white blood cell count, increased cerebrospinal fluid protein, and decreased cerebrospinal fluid glucose; abnormal peripheral blood cell counts, such as high or low white cell count, low platelet count, etc. On day 16, taking the result of NGS cerebrospinal fluid and Sputum culture, Clinical signs, Laboratory tests, including the cerebrospinal fluid test results, we diagnosed the patient with purulent meningitis.

Supportive treatment of neonatal purulent meningitis includes adequate oxygenation, prevention of hypoglycemia, effective anti-epileptic treatment, control of increased intracranial pressure, and nutritional support. In China, in the anti-infective treatment of neonatal purulent meningitis, ampicillin combined with cefotaxime is usually used for early onset infection (0-6 days old). Ampicillin combined with third-generation cephalosporin are usually used for late-onset (acquired after age six days) for newborn from community, Newborns who have been inpatient since birth, vancomycin combined with third-generation Cephalosporin or Meropenem are usually used (4). For newborns with CRKP-associated purulent meningitis, the choice of antibacterial agents is often limited, and the mortality rate is high. Timely, effective anti-infective treatment is the key to successful treatment. Our evaluation of the risks and benefits of the use of quinolones as anti-infective treatment in newborns shows that when no safe and effective antiinfective alternatives are available, fluoroquinolones combined with other drugs that Klebsiella pneumoniae are susceptible to can reduce the mortality risk of newborns with purulent meningitis caused by carbapenem-resistant gram-negative bacteria. However, the patients must be monitored for adverse drug reactions during treatment. Moreover, the patients should be followed up after discharge to monitor their long-term outcomes. The patients also need to undergo regular X-ray or magnetic 


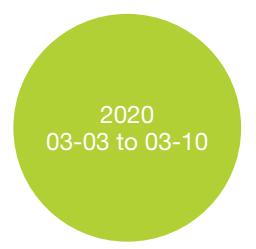

Meropenem $0.07 \mathrm{~g}$ (20 mg/kg), ivgtt, q8h
Vancomycin was added at the dose of $0.04(10 \mathrm{mg} / \mathrm{kg})$, ivgtt,

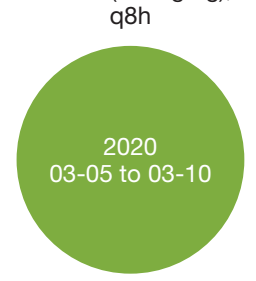

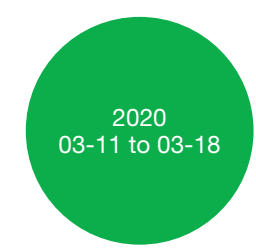

Meropenem and vancomycin were discontinued; acyclovir $0.06 \mathrm{~g}(15 \mathrm{mg} / \mathrm{kg})$, ivgtt, q8h; methylprednisolone sodium succinate $4 \mathrm{mg}(1 \mathrm{mg} / \mathrm{kg})$, ivgtt, qd

Figure 1 Timeline of drug treatment.

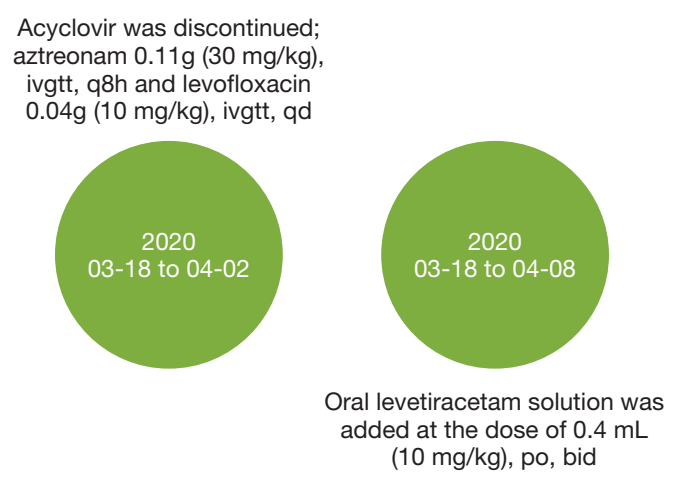

(10 mg/kg), po, bid resonance imaging (MRI) of the bones and joints to detect any long-term quinolone-related musculoskeletal adverse events.

\section{Drug resistance mechanism of carbapenem-resistant Enterobacteriaceae (CRE)}

The mechanism of CRE is related to its molecular diversity, including porin changes in plasmid-mediated Klebsiella pneumoniae carbapenemase (KPC) and metallo$\beta$-lactamases, chromosome-encoded $\beta$-lactamases, and broad-spectrum $\beta$-lactamases (5). The mechanisms of the resistance of Klebsiella pneumoniae to carbapenems include the production of carbapenemases, overexpression of AmpC beta-lactamases combined with loss of outer-membrane porin, and a decreased number, loss, or low affinity of penicillin-binding protein 2 (PBP2), a high-affinity site of carbapenems. Common carbapenemases include (I) class A KPC (KPC-2, KPC-3), (II) class B carbapenemases (metallo- $\beta$-lactamases), and (III) class D carbapenemases (6). The most common CRE is carbapenemase-producing Klebsiella pneumoniae (6). In this report, the patient was infected with CRKP; however, we did not analyze the type of carbapenemases.

\section{Selection of antibiotic agents in children with CRKP}

To treat CRKP, a combination of at least two antibacterial drugs are required based on the results of drug sensitivity, but the optimal combination of antibacterial drugs is currently uncertain. The reasons of combination therapy include, severe CRKP infection has a higher mortality rate, evidence suggests that the combined treatment plan is related to reducing mortality, and worry about drug resistance in monotherapy (7).

The preferred choices, based on drug susceptibility tests, are polymyxin or tigecycline combined with aminoglycosides, fluoroquinolones, minocycline, and sulfamethoxazole-trimethoprim (8). Polymyxin is not recommended in children because it may cause kidney injury. Tigecycline and minocycline may cause permanent discoloration of a child's teeth. Aminoglycosides have significant ototoxicity and nephrotoxicity. Sulfamethoxazole-trimethoprim may cause liver and kidney, thrombocytopenia, aplastic anemia, and kernicterus and is therefore contraindicated in newborns and infants under 2 months of age. The 2017 Infectious Diseases Society of America's Clinical Practice Guidelines for HealthcareAssociated Ventriculitis and Meningitis recommends the use of aztreonam or ciprofloxacin for the treatment of gramnegative bacterial infection (strong, moderate) in patients who are allergic to $\beta$-lactams or are contraindicated for meropenem (9).

In this report, the patient was a late newborn (22-dayold). She was diagnosed with purulent meningitis and neonatal pneumonia. She had intermittent high fever with high-risk factors for CRKP, such as intravenous nutrition, assisted ventilation, and long-term use of broad-spectrum antibiotics $(10,11)$. Before admission, the patient had an episode of mottled skin after administration of cefoperazone sodium and sulbactam sodium at another hospital, which may suggest cephalosporin-related anaphylactic shock. During hospitalization, her systemic rashes worsened after administration of meropenem, which may suggest a meropenem allergy. Based on drug susceptibility tests and the fact that levofloxacin and aztreonam can easily 
cross the blood-brain barrier, the clinical pharmacist recommended anti-infective treatment with levofloxacin combined with aztreonam and for the patient to be closely monitored for any change in vital signs. After one week of treatment, infection-related indicators, routine analysis and biochemical analysis of cerebrospinal fluid, and clinical symptoms were significantly improved.

\section{The safety of quinolones in children}

According to the 2019 report from the US Food and Drug Administration (FDA) and the European Medicines Agency (EMA) (12), serious adverse events seen in patients (both adults and children) who used fluoroquinolones such as ciprofloxacin, levofloxacin, and moxifloxacin between 2013 and 2018 included tendon rupture, retinal detachment, hearing loss, QT prolongation, and aortic aneurysm. A randomized, double-blind, prospective, long-term followup clinical study of 2,523 children from 6 months to 16 years of age showed that more musculoskeletal adverse events were observed in the levofloxacin group than in the control group at the 12-month follow-up, but no significant between-group difference in musculoskeletal adverse events was observed at the 5-year follow-up (13). Other studies showed that the incidence and severity of articular cartilage damage were much lower in children than in animals after administration of fluoroquinolones (14). The China Food and Drug Administration (CFDA), the FDA, and the EMA have approved levofloxacin for the treatment of inhalational anthrax (postexposure) in patients 6 months of age or older; the FDA and the EMA have approved ciprofloxacin for the treatment of complicated pyelonephritis and severe and complicated urinary infections in children aged 1 to 17 years; the FDA has approved levofloxacin for the treatment of plague in children 6 months of age or older and adults $(15,16)$. Therefore, after weighing the risks and benefits, it may be suitable for children to use fluoroquinolones. During treatment, the patients should be closely monitored for common, severe, and potential adverse events, such as potential musculoskeletal or neurological events.

In this report, the patient was given levofloxacin $0.04 \mathrm{~g}(10 \mathrm{mg} / \mathrm{kg})$, ivgtt, qd for 16 days. Her symptoms were alleviated, with no apparent neurological, skeletal, joint, tendon, or cardiac adverse events. The patient will need long-term follow-up after discharge to monitor her outcomes and undergo regular X-ray or MRI of the bones and joints to monitor any long-term musculoskeletal adverse events.

\section{Permeability of the blood-brain barrier to quinolones and aztreonam}

To effectively treat intracranial infection, the antibiotic agent's ability to cross the blood-brain barrier and reach the minimum inhibitory concentration (MIC) in the brain is a key consideration, in addition to the antibacterial spectrum and drug susceptibility tests. Most quinolones have a molecular weight of approximately $300 \mathrm{Da}$, with a low plasma protein binding rate (20\% to $40 \%)$, and are moderately lipophilic. They generally do not dissociate in normal cerebrospinal fluid or plasma. In the absence of meningitis, a much higher percentage of quinolones (e.g., up to $71 \%$ for levofloxacin) than $\beta$-lactams crosses the blood-brain barrier, and in the presence of meningitis as well, more than $70 \%$ of quinolones can cross the bloodbrain barrier (17). Pharmacokinetic analysis shows that the cerebrospinal fluid/plasma area under the curve (AUC) ratio is $0.4-0.6$ or higher for levofloxacin (18).

In the cerebrospinal fluid, aztreonam can reach its MIC for most gram-negative bacteria except Acinetobacter baumannii. It can cross the blood-brain barrier well in the case of meningitis. Modai et al. showed that $14.2 \%$ to $18.4 \%$ of aztreonam crossed the blood-brain barrier on days $2-4$ of treatment, and $5.3 \%$ to $22.9 \%$ crossed the blood-brain barrier on days 11-20 of treatment (19). Aztreonam is also relatively stable in the presence of most $\beta$-lactamases and can be used to treat meningitis caused by $\beta$-lactamase-producing bacteria.

In this report, levofloxacin and aztreonam reached high concentrations in the cerebrospinal fluid to achieve satisfactory anti-infective outcomes.

\section{Selection of the levofloxacin dose}

The Pediatric \& Neonatal Dosage Handbook (20) states that for children aged 6 months to 5 years, the recommended oral or intravenous dose of levofloxacin is 8 to $10 \mathrm{mg} / \mathrm{kg}$, $\mathrm{q} 12 \mathrm{~h}$. There is no recommended dose for children under 6 months of age. The Martindale: The extra pharmacopeia (21) states that fluoroquinolones are not absolutely contraindicated in children and adolescents and may be used in these populations after weighing the risks and benefits at the dose of $8 \mathrm{mg} / \mathrm{kg}$ (maximum $250 \mathrm{mg}$ ), q12h. Pharmacokinetic analysis (22) shows that for children over 6 months, the half-life of levofloxacin increases with age. Newborns have different renal function than older infants or children. For example, their glomerular filtration rate and tubular secretion rate are lower, which may slow the clearance of levofloxacin; 
however, no pharmacokinetic studies of levofloxacin have been conducted in newborns.

In this report, the clinical pharmacist recommended levofloxacin $10 \mathrm{mg} / \mathrm{kg} /$ day ivgtt. After one week of treatment, the clinical symptoms were significantly ameliorated. During treatment, the patient's liver and kidney functions were normal. The patient will receive long-term follow-up after discharge, including regular X-ray or MRI of the bones and joints to detect any adverse musculoskeletal reactions, regular transcranial color Doppler ultrasound or MRI to detect any adverse neurological reactions, enhanced care to prevent reinfection, and regular vision screening, hearing screening, and administration of the Denver Developmental Screening Test.

\section{Recommendations for newborn purulent meningitis caused by CRKP}

By participating in the antibacterial drug adjustment and dosage recommendation during the treatment of this case of newborn purulent meningitis, for the drug treatment of children with multidrug-resistant Klebsiella pneumoniae purulent meningitis, clinical pharmacists suggested that choose susceptible antibiotic combination therapy based on the results of drug sensitivity, may reduce mortality and drug resistance in monotherapy. In this newborn, levofloxacin combined with aztreonam was used and the newborn's purulent meningitis was controlled and alleviated. The treatment course of levofloxacin is not easy to be too long, usually 1-2 weeks. During the treatment process, while evaluation the effectiveness of the medication in children, it is also necessary to pay attention to the adverse reactions of the medication, and carry out longterm follow-up, and dynamically adjust the medication according to the treatment effect and the occurrence of adverse reactions. Supportive treatments such as adequate oxygenation, prevention of hypoglycemia, effective antiepileptic treatment, and nutritional support are equally important. It is also need to strengthen hospital infection management, such as timely care, hand hygiene, contact isolation, and environmental cleanliness.

\section{Conclusions}

The clinical pharmacist was involved in the adjustment of antibacterial agents and dosage recommendations during the treatment of purulent meningitis in this patient and played an important role in improving treatment outcomes and promoting clinical safety and rational drug use. Besides effectiveness, it is important to monitor adverse drug reactions and perform long-term followup. Clinical pharmacists should keep clinicians updated on the guidelines for fluoroquinolone use in children. In this paper, we analyzed the drug resistance mechanisms of CRKP, the selection of antibiotic agents, the safety of quinolones in children, the permeability of the blood-brain barrier to quinolones, and the selection of the quinolone dose. Personalized combination therapy enhances treatment outcomes and reduces adverse reactions, especially in patients with resistant bacteria infection.

\section{Acknowledgments}

Funding: Gansu Natural Science Foundation Program (17JR5RA239); Lanzhou Talent Innovation and Entrepreneurship Program (No. 2018-RC-46).

\section{Footnote}

Reporting Checklist: The authors have completed the CARE reporting checklist. Available at http://dx.doi.org/10.21037/ tp-20-296

Conflicts of Interest: All authors have completed the ICMJE uniform disclosure form (available at http://dx.doi. org/10.21037/tp-20-296). The authors have no conflicts of interest to declare.

Ethical Statement: The authors are accountable for all aspects of the work in ensuring that questions related to the accuracy or integrity of any part of the work are appropriately investigated and resolved. All procedures performed in studies involving human participants were in accordance with the ethical standards of the institutional and/or national research committee(s) and with the Helsinki Declaration (as revised in 2013). Written informed consent was obtained from the patient for publication of this study and any accompanying images.

Open Access Statement: This is an Open Access article distributed in accordance with the Creative Commons Attribution-NonCommercial-NoDerivs 4.0 International License (CC BY-NC-ND 4.0), which permits the noncommercial replication and distribution of the article with the strict proviso that no changes or edits are made and the 
original work is properly cited (including links to both the formal publication through the relevant DOI and the license). See: https://creativecommons.org/licenses/by-nc-nd/4.0/.

\section{References}

1. Gaschignard J, Levy C, Romain O, et al. Neonatal Bacterial Meningitis: 444 Cases in 7 Years. Pediatr Infect Dis J 2011;30:212.

2. Vock I, Tschudin-Sutter S. Carbapenem-resistant Klebsiella pneumoniae-impact of infection-prevention and control interventions. Ann Transl Med 2019;7:S344.

3. Nizet V, Klein JO. Bacterial sepsis and meningitis. In: Infectious Diseases of the Fetus and Newborn Infant, 8th ed. In: Remington JS, Klein JO, Wilson CB, et al. editors. Philadelphia: Elsevier Saunders, 2016:217.

4. Saez-Llorens X, McCracken GH Jr. Krugman's Infectious Diseases of Children. In: Krugman's Infectious Diseases of Children, 11th ed. In: Greshon AA, Hotez PJ, Katz SL. editors. Philadelphia: Mosby, 2004:373.

5. Little ML, Qin X, Zerr M, et al. Molecular diversity in mechanisms of carbapenem resistance in paediatric Enterobacteriaceae. Int J Antimicrob Agents 2012;39:52-7.

6. Montagnani C, Prato M, Scolfaro C, et al. Carbapenemresistant Enterobacteriaceae Infections in Children: An Italian Retrospective Multicenter Study. Pediatr Infect Dis J 2016;35:862-8.

7. Qureshi ZA, Paterson DL, Potoski BA, et al. Treatment outcome of bacteremia due to KPC-producing Klebsiella pneumoniae: superiority of combination antimicrobial regimens. Antimicrob Agents Chemother 2012;56:2108.

8. World Health Organization. Guidelines for the prevention and control of carbapenem-resistant Enterobacteriaceae, Acinetobacter baumannii and Pseudomonas aeruginosa in health care facilities. (2018-02-23) [2018-03-01]. Available online: http://www.who.int/infectionprevention/ publications/guidelines-cre/en/

9. Tunkel AR, Hasbun R, Bhimraj A, et al. 2017 Infectious Diseases Society of America's Clinical Practice Guidelines for Healthcare-Associated Ventriculitis and Meningitis. Clin Infect Dis 2017;64:e34-65.

10. Montagnani C, Prato $M$, Scolfaro C, et al. Carbapenem-resistant enterobacteriaceae infections in children: An Italian retrospective multicenter study. Pediatr Infect Dis J 2016;35:862-8.

11. Goodman KE, Simner PJ, Tamma PD, et al. Infection control implications of heterogeneous resistance mechanisms in carbapenem-resistant Enterobacteriaceae
(CRE). Expert Rev Anti Infect Ther 2016;14:95-108.

12. Bennett AC, Bennett CL, Witherspoon BJ, et al. An evaluation of reports of ciprofloxacin, levofloxacin, and moxifloxacin-association neuropsychiatric toxicities, longterm disability, and aortic aneurysms/dissections disseminated by the Food and Drug Administration and the European Medicines Agency. Expert Opin Drug Saf 2019;18:1055-63.

13. Bradley JS, Kauffman RE, Balis DA, et al. Assessment of musculoskeletal toxicity 5 years after therapy with levofloxacin. Pediatrics 2014;134:e146-53.

14. Bacci C, Galli L, de Martino M, et al. Fluoroquinolones in children: update of the literature. J Chemother 2015;27:257-65.

15. Wright JG, Quinn CP, Shadomy S, et al. Use of anthrax vaccine in the United States: recommendations of the Advisory Committee on Immunization Practices (ACIP), 2009. MMWR Recomm Rep 2010;59:1-30.

16. Gupta K, Hooton TM, Naber KG, et al. International clinical practice guidelines for the treatment of acute uncomplicated cystitis and pyelonephritis in women: a 2010 update by the Infectious Diseases Society of America and the European Society for Microbiology and Infectious Diseases. Clin Infect Dis 2011;52:e103-20.

17. Nau R, Seele J, Djukic M, et al. Pharmacokinetics and pharmacodynamics of antibiotics in central nervous system infections. Curr Opin Infect Dis 2018;31:57-68.

18. Alffenaar JW, van Altena R, Bökkerink HJ, et al. Pharmacokinetics of moxifloxacin in cerebrospinal fluid and plasma in patients with tuberculous meningitis. Clin Infect Dis 2009;49:1080-2.

19. Modai J, Vittecoq D, Decazes JM, et al. Penetration of aztreonam into cerebrospinal fluid of patients with bacterial meningitis. Antimicrob Agents Chemother 1986;29:281-3.

20. Taketomo CK, Hodding JH, Kraus DM. American Pharmacist Association. Pediatric \& Neonatal Dosage Handbook. 24th ed. Lexicomp, 2016.

21. James EF, Reynolds. MARTINDALE the extra pharmacopoeia. 38th. London: Royal Pharmaceutical Society, 2014;316.

22. Chien S, Wells TG, Blumer JL, et al. Levofloxacin pharmacokinetics in children. J Clin Pharmacol 2005; 45: 153-60.

Cite this article as: Wang F, Jiang J, Shi G, Wang J, Zhou S. Anti-infective treatment of purulent meningitis caused by carbapenem-resistant Klebsiella pneumoniae in a newborn: a case report. Transl Pediatr 2020;9(5):713-719. doi: 10.21037/tp-20-296 